without the e4 allele had died by December 2008. Median survival from onset of AD did not differ by the e4 allele carrier status (10.2 years in the e4positive group, 9.1 years in the e4-negative group). There were no differences between e4-positive and e4-negative group in age at baseline, sex, education, age at onset, duration of $\mathrm{AD}$, severity of dementia. Significant factors that affects mortality during the follow-up included age at baseline and age at onset. Adjusting for age at baseline and age at onset, the presence of an e4 allele did not show increased risk of mortality $(\mathrm{RR}=0.98,95 \%$ $\mathrm{CI}=0.64-1.49)$ and the risk of effect of the e4 allele not vary by age, sex, education in this sample. Conclusions: This study provide the first information about the effect of the e 4 allele on mortality in oriental elderly with AD. The APOE e4 allele was not associated with mortality in patients with $\mathrm{AD}$.

\section{P1-241 IMPACT OF APOE GENOTYPE DISCLOSURE ON EXERCISE AND MENTAL ACTIVITIES}

Erin Klopfenstein ${ }^{1}$, Clara Chen ${ }^{1}$, Kurt Christensen ${ }^{2}$, Denise Lautenbach ${ }^{1}$, L. Adrienne Cupples ${ }^{1}$, Scott Roberts ${ }^{2}$, Robert Green ${ }^{1},{ }^{1}$ Boston University, Boston, Massachusetts, United States; ${ }^{2}$ University of Michigan School of Public Health, Ann Arbor, Michigan, United States.

Background: The impact of genetic susceptibility information about Alzheimer's disease (AD) on exercise and mental activities is unclear. The Risk Evaluation and Education for Alzheimer's Disease (REVEAL) Study is a series of multi-site randomized controlled clinical trials examining the impact of Apolipoprotein $\mathrm{E}(A P O E)$ genetic risk disclosure for AD. We hypothesized that $A P O E$ e 4 carriers are more likely than non-carriers to report changes to their exercise and mental activities after disclosure. Methods: 451 subjects with affected first-degree relatives in the second and third REVEAL trials received AD risk assessments based on APOE genotype, family history, ethnicity and gender (risk range: $13-74 \%$ ). Six weeks and 12 months after the risk disclosure session, subjects were asked yes-no questions regarding changes to exercise plans or mental activities to help prevent $\mathrm{AD}$. Logistic regression was used to examine whether e4 carriers were more likely than non-carriers to report behavior changes at either time point after adjusting for age, gender, race, education and randomization arm. > Results: $43 \%$ of e4 carriers reported changing mental activities by the 12-month follow-up session, compared to $32 \%$ of non-carriers. $40 \%$ of e 4 carriers reported changing exercise plans by the 12-month follow-up session, compared to $35 \%$ of non-carriers. Logistic regression showed that e 4 carriers were more likely to report a change in mental activities than non-carriers $(\mathrm{OR}=1.60, \mathrm{p}=0.02)$, but not necessarily more likely to report a change in physical exercise $(O R=1.31, p=0.17)$. Conclusions: Learning about increased risk for $\mathrm{AD}$ due to $A P O E$ genotype motivates people to make changes to mental activities. Neither mental activities or exercise are proven $\mathrm{AD}$ prevention strategies, but both are promoted as possible ways of reducing risk, which may explain why $40 \%$ or more of e 4 carriers reported changes in these behaviors.

\section{P1-242 COMPREHENSIVE VARIANT DISCOVERY IN THE LATE-ONSET ALZHEIMER'S DISEASE SUSCEPTIBILITY GENE MTHFD1L USING NEXT GENERATION SEQUENCING TECHNOLOGY}

Martin Kohli ${ }^{1}$, Adam $\mathrm{Naj}^{1}$, Jessica Van Baaren ${ }^{1}$, William Hulme ${ }^{1}$, Gary Beecham ${ }^{1}$, Joseph Buxbaum ${ }^{2}$, Stephan Zuchner ${ }^{1}$, Jonathan Haines ${ }^{3}$, John Gilbert ${ }^{1}$, Margaret Pericak-Vance ${ }^{1},{ }^{1}$ John P Hussman Institute for Human Genomics, University of Miami, Miami, Florida, United States; ${ }^{2}$ Mount Sinai Medical Center, New York, New York, United States; ${ }^{3}$ Vanderbilt University Medical Center, Nashville, Tennessee, United States.

Background: We identified and replicated the association of MTHFD1L intronic SNP rs11754661 with Late-onset Alzheimer's disease (LOAD) with genome-wide statistical significance in a previous genome-wide association study ( $P=1.90 \tilde{\mathrm{A}}-10^{-10}$ in combined datasets). MTHFD1L is involved in the folate pathway and represents an interesting biological LOAD candidate since it may influence homocysteine levels, a significant risk factor for LOAD. rs11754661 is located in a region of high linkage disequilibrium (LD) extending for $32.6 \mathrm{~kb}$ of the gene's $360.7 \mathrm{~kb}$ length. We resequenced this LD block to identify and characterize rare and potentially functional variants which may contribute to the observed association. Methods: To pinpoint rare and potentially functional variants, we performed comprehensive re-sequencing using Illumina's Genome Analyzer in 50 LOAD cases and 34 age-matched cognitively normal controls. Long-range PCR on samples' genomic DNA targeted a $50 \mathrm{~kb}$ region comprising the entire LD block containing the previously associated SNP plus flanking sequence. Sequence read alignment and variant calling was performed using Maq. Characterizing LD with variant data from sequencing, we pooled rare variants within $8 \mathrm{LD}$ blocks and used the Cochran-Armitage trend test to test association. Results: 129 SNPs were detected (82 were novel, 47 previously annotated in dbSNP131). Excluding one novel synonymous coding variant observed in one control, all SNPs were intronic or mapped 5' of MTHFD1L. 24 SNPs were novel and common (MAF $>0.05)$. Most novel SNPs (52/82) were rare variants, with 36 unique to cases and 22 unique to controls. Nine common SNPs showed medium-to-high LD with rs11754661, of which two localized to the 5' untranslated region of a specific isoform of MTHFD1L. Examining rare variant associations by pooling, we observed a strong association among variants with $\mathrm{MAF}<0.05$ in $\mathrm{LD}$ block $5(\mathrm{P}=0.00011)$, though this block is adjacent to the LD block 6 containing rs11754661. Conclusions: Re-sequencing of the MTHFDIL LD block containing rs11754661 identified novel variants, some of interest for further fine-mapping of the association. Though obvious functional SNPs which might explain the association have not yet been identified, pooled rare variants in a narrowed region of LD demonstrated evidence of association with LOAD, suggesting a need for further examination of rare variants in this region.

\section{P1-243 TRISOMY FOR SYNAPTOJANIN1 IN DOWN'S SYNDROME IS FUNCTIONALLY LINKED TO THE ENLARGEMENT OF EARLY ENDOSOMES, THE FIRST MORPHOLOGICAL EVIDENCE OF ALZHEIMER'S DISEASE}

Jeremie Lavaur ${ }^{1}$, Jack Cossec ${ }^{2}$, Alexander Hoischen ${ }^{3}$, Samantha Stora ${ }^{4}$, Magalie Lecourtois ${ }^{5}$, Clotilde Mircher ${ }^{4}$, Yann Grattau,

Charles Duyckaerts ${ }^{2}$, Stylianos Antonarakis ${ }^{6}$, Joris Veltman ${ }^{3}$, JeanChristophe Olivo Marin ${ }^{7}$, Fabrice de Chaumont ${ }^{8}$, Clémentine Ripoll ${ }^{9}$, Jean Delabar ${ }^{9}$, Gilberto di Paolo ${ }^{10}$, Marie-Claude Potier ${ }^{2},{ }^{1}$ CRICM, Paris, France; ${ }^{2}$ Centre de Recherche de l'Institut du Cerveau et de la Moelle épinière UPMC/Inserm UMR-S 975; CNRS UMR 7225, Paris, France; ${ }^{3}$ Radboud University, Nijmegen, Netherlands; ${ }^{4}$ Institut Jérôme Lejeune, paris, France; ${ }^{5}$ InsermU614, IFRMP23, Rouen Institute for Medical Research and Innovation, Faculty of Medicine, University of Rouen, Rouen, France; ${ }^{6}$ Department of Genetic Medicine and Development, University of Geneva Medical School and University Hospitals of Geneva, Geneva, Switzerland, Geneva, Switzerland; ${ }^{7}$ Institut Pasteur, paris, France; ${ }^{8}$ Institut Pasteur, Paris, France $;{ }^{9}$ Unité de biologie fonctionnelle et adaptative (BFA), EAC CNRS 4413, Université Paris Diderot, Marie-Andrée Lagroua-Weill-Hallé, bÃ ctiment Lamarck, Paris, France; ${ }^{10}$ Department of Pathology and Cell Biology, Taub Institute for Research on Alzheimer's Disease and the Aging Brain, Columbia University Medical Center, New York, New York, United States.

Enlarged endosomes are the first morphological change observed in the brain of Alzheimer's disease and Down's syndrome individuals. Here we demonstrate their presence in blood mononuclear and lymphoblastoid cells lines (LCLs) from Down's syndrome individuals. In LCLs carrying APP microduplications enlarged endosomes were absent, suggesting that APP overexpression is not involved in the enlargement of early endosomes in this cell type. Using genotype-phenotype correlations in LCLs carrying partial trisomies 21 we have demonstrated that triplication of a $1.3 \mathrm{Mb}$ locus from chromosome 21 , containing the synaptojanin 1 gene, is associated with the enlargement of early endosomes. We showed that SYNJ1 transcripts are 